TRANSACTIONS OF THE

AMERICAN MATHEMATICAL SOCIETY

Volume 353, Number 11, Pages 4357-4369

S 0002-9947(01)02814-8

Article electronically published on June 21, 2001

\title{
ON THE SEMISIMPLICITY CONJECTURE AND GALOIS REPRESENTATIONS
}

\author{
LEI FU
}

\begin{abstract}
The semisimplicity conjecture says that for any smooth projective scheme $X_{0}$ over a finite field $\mathbf{F}_{q}$, the Frobenius correspondence acts semisimply on $H^{i}\left(X \otimes_{\mathbf{F}_{q}} \mathbf{F}, \overline{\mathbf{Q}}_{l}\right)$, where $\mathbf{F}$ is an algebraic closure of $\mathbf{F}_{q}$. Based on the works of Deligne and Laumon, we reduce this conjecture to a problem about the Galois representations of function fields. This reduction was also achieved by Laumon a few years ago (unpublished).
\end{abstract}

\section{INTRODUCTION}

Let $\mathbf{F}_{q}$ be a finite field of characteristic $p$ with $q$ elements. Choose an algebraic closure $\mathbf{F}$ of $\mathbf{F}_{q}$. Throughout this paper, schemes, morphisms and sheaves defined on the base field $\mathbf{F}_{q}$ are denoted by letters with subscripts 0 and we indicate the base extension from $\mathbf{F}_{q}$ to $\mathbf{F}$ by dropping the subscripts 0 . But for convenience, the projective line over $\mathbf{F}_{q}$ and over $\mathbf{F}$ are both denoted by $\mathbf{P}^{1}$, and the affine line over $\mathbf{F}_{q}$ and over $\mathbf{F}$ are both denoted by $\mathbf{A}^{1}$. Schemes and morphisms are separated and of finite type.

Let $X_{0}$ be a scheme over $\mathbf{F}_{q}$, let $\mathcal{F}_{0}$ be a constructible $\overline{\mathbf{Q}}_{l}$-sheaf on $X_{0}$, and let $\iota: \overline{\mathbf{Q}}_{l} \rightarrow \mathbf{C}$ be an isomorphism. Recall that $\mathcal{F}_{0}$ is called puncturely $\iota$-pure with weight $w$ if for every closed point $x$ of $X_{0}$ and for every eigenvalue $\lambda$ of the geometric Frobenius automorphism $F_{x}$ on $\mathcal{F}_{\bar{x}}$, the absolute value of $\iota(\lambda)$ is $N(x)^{w / 2}$, where $N(x)$ is the number of elements of the residue field $k(x)$. Also recall that giving a lisse $\overline{\mathbf{Q}}_{l}$-sheaf on a connected scheme is the same as giving a $\overline{\mathbf{Q}}_{l}$-representation of the fundamental group of the scheme. So we can talk about the irreducibility and the semisimplicity of a lisse sheaf.

If $X_{0}$ is a normal geometrically connected scheme over $\mathbf{F}_{q}$ and if $\mathcal{F}_{0}$ is a puncturely $\iota$-pure lisse $\overline{\mathbf{Q}}_{l}$-sheaf on $X_{0}$, then by a theorem of Deligne (D1], 3.4.1 (iii)), $\mathcal{F}$ is a semisimple sheaf on $X$. In $[\underline{F}$, based on Deligne's work, we get the following result:

Theorem 1. Let $X_{0}$ be a normal geometrically connected scheme of finite type over $\mathbf{F}_{q}$ and let $\mathcal{F}_{0}$ be a puncturely ı-pure lisse $\overline{\mathbf{Q}}_{l}$-sheaf on $X_{0}$. Fix a geometric point $\bar{x}$ in $X_{0}$. If there exists an element in the Weil group $W\left(X_{0}, \bar{x}\right)$ but not in the geometric fundamental group $\pi_{1}(X, \bar{x})$ such that its action on $\mathcal{F}_{\bar{x}}$ is semisimple, then $\mathcal{F}_{0}$ is a semisimple sheaf on $X_{0}$.

Received by the editors November 5, 1999.

1991 Mathematics Subject Classification. Primary 14F20, 14G15.

Key words and phrases. $F$-semisimple representations, puncturely pure sheaves, $l$-adic Fourier transformations, perverse sheaves. 
Here a linear transformation on a vector space is said to be semisimple if the corresponding matrix is diagonalizable. In $[\mathbf{F}$, the element in the Weil group but not in the geometric fundamental group is taken to be a Frobenius automorphism. But the proof works in the general case.

Let $K$ be a local field with finite residue field, let $W(\bar{K} / K)$ be its Weil group and let $I$ be its inertia group. We say a $\overline{\mathbf{Q}}_{l}$-representation $\operatorname{Gal}(\bar{K} / K) \rightarrow \operatorname{GL}(V)$ is $F$-semisimple if every element in $W(\bar{K} / K)-I$ acts semisimply on $V$. For more details about this concept, see [D2] $\S 8$, or Lemma 1.1 of this paper.

Let $S_{0}$ be a smooth geometrically connected curve over $\mathbf{F}_{q}$ and let $\bar{S}_{0}$ be its smooth compactification. For every closed point $x$ in $\bar{S}_{0}$, let $\eta_{x}$ be the generic point of the henselization of $\bar{S}_{0}$ at $x$, and let $\bar{\eta}_{x}$ be a geometric generic point of the strict henselization of $\bar{S}_{0}$ at $\bar{x}$. Then for any lisse $\overline{\mathbf{Q}}_{l}$-sheaf $\mathcal{F}_{0}$ on $X_{0}$, the stalk $\mathcal{F}_{\bar{\eta}_{x}}$ is a representation of the Galois group $\operatorname{Gal}\left(\bar{\eta}_{x} / \eta_{x}\right)$. We say $\mathcal{F}_{0}$ is locally semisimple if $\mathcal{F}_{\bar{\eta}_{x}}$ is an $F$-semisimple representation of $\operatorname{Gal}\left(\bar{\eta}_{x} / \eta_{x}\right)$ for every closed point $x$ in $\bar{S}_{0}$. By Theorem 1, a puncturely $\iota$-pure locally semisimple lisse $\overline{\mathbf{Q}}_{l}$-sheaf is semisimple. We conjecture that a puncturely $\iota$-pure semisimple sheaf is locally semisimple. Let $\mathcal{F}_{0}$ be a puncturely $\iota$-pure lisse $\overline{\mathbf{Q}}_{l}$-sheaf on $S_{0}$. If $\mathcal{F}_{\bar{\eta}_{x}}$ is $F$-semisimple for one closed point $x$ in $\bar{S}_{0}$, then $\mathcal{F}_{0}$ is a semisimple sheaf on $S_{0}$ by Theorem 1 . If our conjecture is true, then $\mathcal{F}_{\bar{\eta}_{y}}$ is $F$-semisimple for every closed point $y$ in $\bar{S}_{0}$. Let's state a special case of this conjecture:

Main Conjecture. Let $\mathcal{F}_{0}$ be a puncturely $\iota$-pure lisse $\overline{\mathbf{Q}}_{l}$-sheaf on $\mathbf{P}^{\mathbf{1}}-\{0, \infty\}$. If $\mathcal{F}_{\bar{\eta}_{\infty}}$ is $F$-semisimple, then $\mathcal{F}_{\bar{\eta}_{0}}$ is $F$-semisimple.

In the language of algebraic number theory, this conjecture says that if $V$ is a $\iota$-pure $\overline{\mathbf{Q}}_{l}$-representation of $\operatorname{Gal}\left(\overline{\mathbf{F}_{q}(t)} / \mathbf{F}_{q}(t)\right)$ unramified outside 0 and $\infty$ such that $V$ is an $F$-semisimple representation for $\operatorname{Gal}\left(\overline{\mathbf{F}_{q}\left(\left(\frac{1}{t}\right)\right)} / \mathbf{F}_{q}\left(\left(\frac{1}{t}\right)\right)\right)$, then $V$ is an $F$-semisimple representation for $\operatorname{Gal}\left(\overline{\mathbf{F}_{q}((t))} / \mathbf{F}_{q}((t))\right)$, where $\mathbf{F}_{q}((t))$ is the field of formal Laurent series.

Recall that Langlands' program says that semisimple Galois representations of global fields correspond to automorphic representations, and $F$-semisimple Galois representations of local fields correspond to admissible representations. On the other hand, automorphic representations are restricted tensor products of admissible representations. So once the compatibility of local and global Langlands correspondences is established, our conjecture follows.

In $\S 1$, inspired by Laumon's proof of the Weil conjecture $([\overline{\mathrm{L}}])$, we prove the following theorem using $l$-adic Fourier transformations:

Theorem 2. Assume the main conjecture holds. Let $S_{0}$ be a smooth geometrically connected curve over $\mathbf{F}_{q}, \bar{S}_{0}$ the smooth compactification of $S_{0}$, and $j: S_{0} \rightarrow$ $\bar{S}_{0}$ the open immersion. Then for any lisse locally semisimple puncturely ı-pure $\overline{\mathbf{Q}}_{l}$-sheaf $\mathcal{F}_{0}$ on $S_{0}$, the geometric Frobenius correspondence $F$ acts semisimply on $H^{i}\left(\bar{S}, j_{*} \mathcal{F}\right)$.

Corollary. Assume the main conjecture holds. Let $S_{0}$ be a smooth geometically connected curve over $\mathbf{F}_{q}$. Then for any lisse locally semisimple puncturely ı-pure $\overline{\mathbf{Q}}_{l}$-sheaf $\mathcal{F}_{0}$ on $S_{0}$, the geometric Frobenius correspondence $F$ acts semisimply on $H_{c}^{i}(S, \mathcal{F})$. 
Proof. Let $\bar{S}_{0}$ be the smooth compactification of $S_{0}$ and $j: S_{0} \rightarrow \bar{S}_{0}$ the open immersion. We have a long exact sequence

$$
\begin{array}{rlllc}
0 & \rightarrow H_{c}^{0}(S, \mathcal{F}) & \rightarrow H^{0}\left(\bar{S}, j_{*} \mathcal{F}\right) & \rightarrow & H^{0}\left(\bar{S}-S,\left.\left(j_{*} \mathcal{F}\right)\right|_{\bar{S}-S}\right) \\
& \rightarrow H_{c}^{1}(S, \mathcal{F}) & \rightarrow H^{1}\left(\bar{S}, j_{*} \mathcal{F}\right) & \rightarrow & 0 \\
& \rightarrow H_{c}^{2}(S, \mathcal{F}) & \rightarrow H^{2}\left(\bar{S}, j_{*} \mathcal{F}\right) & \rightarrow & 0
\end{array}
$$

This exact sequence and Theorem 2 show immediately that $F$ acts semisimply on $H_{c}^{0}(S, \mathcal{F})$ and on $H_{c}^{2}(S, \mathcal{F})$. Since $\mathcal{F}_{0}$ is locally semisimple, for any $x \in \bar{S}_{0}-S_{0}, \mathcal{F}_{\bar{\eta}_{x}}$ is an $F$-semisimple representation of $\operatorname{Gal}\left(\bar{\eta}_{x} / \eta_{x}\right)$. Let $I$ be the inertia subgroup of $\operatorname{Gal}\left(\bar{\eta}_{x} / \eta_{x}\right)$. Since $I$ is a normal subgroup, $\mathcal{F}_{\bar{\eta}_{x}}^{I}=\left(j_{*} \mathcal{F}\right)_{\bar{x}}$ is a semisimple representation $\operatorname{Gal}\left(\bar{\eta}_{x} / \eta_{x}\right) / I \cong \operatorname{Gal}(\overline{k(x)} / k(x))$. So $F_{x}$ acts semisimply on $\left(j_{*} \mathcal{F}\right)_{\bar{x}}$. Let $w$ be the weight of $\mathcal{F}_{0}$. By D1 1.8.1, the weights of $F_{x}$ on $\left(j_{*} \mathcal{F}\right)_{\bar{x}}$ are $\leq w$. So $F$ acts semisimply on $H^{0}\left(\bar{S}-S,\left(j_{*} \mathcal{F}\right)_{\bar{S}-S}\right)$ with weights $\leq w$. By [D1] $3.2 .3, F$ acts purely on $H^{1}\left(\bar{S}, j_{*} \mathcal{F}\right)$ with weight $w+1$ and by Theorem 2 this action is semisimple. The above long exact sequence then shows that $F$ acts semisimply on $H_{c}^{1}(S, \mathcal{F})$.

In $\S 2$, we prove the following result using Theorem 2:

Theorem 3. If the main conjecture holds, then the semisimplicity conjecture holds, that is, for any smooth projective scheme $X_{0}$ over $\mathbf{F}_{q}$, the geometric Frobenius correspondence $F$ acts semisimply on $H^{i}\left(X, \overline{\mathbf{Q}}_{l}\right)$.

The semisimplicity conjecture is proved to be true for curves, abelian varieties, K3 surfaces and unirational 3-folds, and it follows from the standard conjectures. We hope that Theorem 3 will point out a new way to approach the semisimplicity conjecture.

G. Laumon also obtained the above results a few years ago (unpublished).

Acknowledgements. The research is supported by the China Science Foundation for Postdoctors, by the Scientific Research Foundation for Returned Overseas Chinese Scholars, and by the Morningside Center of Mathematics at the Chinese Academy of Science.

\section{Proof of Theorem 2}

We start with some lemmas.

Lemma 1.1. Let $K$ be a local field with finite residue field, $W(\bar{K} / K)$ its Weil group, and $I$ its inertia group.

(i) Let $\rho: \operatorname{Gal}(\bar{K} / K) \rightarrow \mathrm{GL}(V)$ be a $\overline{\mathbf{Q}}_{l}$-representation. If there exists one element in $W(\bar{K} / K)-I$ acting semisimply on $V$, then every element in $W(\bar{K} / K)-$ $I$ acts semisimply on $V$, that is, $\rho$ is $F$-semisimple.

(ii) The representation $\rho$ is semisimple if and only if $\rho$ is F-semisimple and $\rho(I)$ is finite.

(iii) Let $L$ be a finite extension of $K$ contained in $\bar{K}$. Then $\rho$ is $F$-semisimple if and only if the restriction of $\rho$ to $\mathrm{Gal}(\bar{K} / L)$ is an $F$-semisimple representation of $\operatorname{Gal}(\bar{K} / L)$.

(iv) Let $\rho_{0}: \operatorname{Gal}(\bar{K} / L) \rightarrow \mathrm{GL}(V)$ be an $F$-semisimple representation. Then the representation $\operatorname{Ind}_{\operatorname{Gal}(\bar{K} / L)}^{\operatorname{Ga}(\bar{K})}\left(\rho_{0}\right)$ of $\operatorname{Gal}(\bar{K} / K)$ induced by $\rho_{0}$ is F-semisimple. 
Proof. (i) By a theorem of Grothendieck ([ST] Appendix), there exists a nilpotent transformation $N: V(1) \rightarrow V$ and a subgroup $I_{1}$ of $I$ with finite index such that for any $\sigma \in I_{1}$, we have $\rho(\sigma)=\exp \left(t_{l}(\sigma) . N\right)$, where $t_{l}: I \rightarrow \mathbf{Z}_{l}(1)$ is defined by $t_{l}(\sigma)=\left(\sigma\left(\pi^{1 / l^{n}}\right) / \pi^{1 / l^{n}}\right)$ for any uniformizer $\pi$ of the local field. We may choose $I_{1}$ so that it is normal in $\operatorname{Gal}(\bar{K} / K)$. Moreover, $N$ commutes with the action of $W(\bar{K} / K)$ on $V(1)$ and on $V$. Fix an element $F \in W(\bar{K} / K)$ with degree 1 . Then every element in $W(\bar{K} / K)$ can be uniquely written as $F^{n} \sigma$ for some $n \in \mathbf{Z}$ and $\sigma \in I$. Define $\rho^{\prime}: W(\bar{K} / K) \rightarrow \mathrm{GL}(V)$ by $\rho^{\prime}\left(F^{n} \sigma\right)=\rho\left(F^{n} \sigma\right) \exp \left(-t_{l}(\sigma) . N\right)$. One can show that $\rho^{\prime}$ is a representation. By the choice of $N$, the representation $\rho^{\prime}$ is trivial on $I_{1}$. In particular, $\rho^{\prime}(I)=\rho^{\prime}\left(I / I_{1}\right)$ is finite.

One can show $\rho\left(F^{n} \sigma\right) \exp \left(-t_{l}(\sigma) \cdot N\right)=\exp (-\mu \cdot N) \rho\left(F^{n} \sigma\right) \exp (\mu \cdot N)$ for $\mu=$ $\frac{t_{l}(\sigma)}{q^{n}-1}$. Hence $\rho^{\prime}\left(F^{n} \sigma\right)$ and $\rho\left(F^{n} \sigma\right)$ are conjugate to each other. In particular, $\rho\left(F^{n} \sigma\right)$ is semisimple if and only if $\rho^{\prime}\left(F^{n} \sigma\right)$ is semisimple.

By assumption, there exist some nonzero integer $n_{0}$ and some $\sigma_{0} \in I$ such that $\rho\left(F^{n_{0}} \sigma_{0}\right)$ is semisimple. Hence $\rho^{\prime}\left(F^{n_{0}} \sigma_{0}\right)$ is also semisimple. For convenience put $g=F^{n_{0}} \sigma_{0}$. Let $h$ be an arbitrary element in $W(\bar{K} / K)-I$. We need to show $\rho(h)$ is semisimple, or equivalently, $\rho^{\prime}(h)$ is semisimple. Since $I / I_{1}$ is finite, there exists some positive integer $m$ such that the automorphism of $I / I_{1}$ induced by $\sigma \mapsto h^{-m} \sigma h^{m}$ is identity. For example we can take $m=\left|\operatorname{Aut}\left(I / I_{1}\right)\right|$.

Let $W^{\prime}$ be the subgroup of $W(\bar{K} / K)$ generated by $I$ and $h$. Since $h$ does not lie in $I$, the subgroup $W^{\prime}$ has finite index in $W(\bar{K} / K)$. We have an exact sequence

$$
0 \rightarrow I / I_{1} \rightarrow W^{\prime} / I_{1} \stackrel{\operatorname{deg}}{\rightarrow} \mathbf{Z} \rightarrow 0
$$

where $\operatorname{deg}$ is defined by $\operatorname{deg}\left(h^{k} \sigma\right)=k$ for any integer $k$ and any $\sigma \in I$. The image of $h^{m}$ in $W^{\prime} / I_{1}$ lies in the center since $W^{\prime} / I_{1}$ is generated by $I / I_{1}$ and the image of $h$, and the image of $h^{m}$ commutes both with elements in $I / I_{1}$ and with the image of $h$. The representation $\rho^{\prime}: I / I_{1} \rightarrow \mathrm{GL}(V)$ is semisimple since $I / I_{1}$ is finite. Moreover since $W^{\prime}$ has finite index in $W(\bar{K} / K)$, some power $g^{n}$ of $g$ lies in $W^{\prime}$. By assumption $\rho^{\prime}\left(g^{n}\right)$ is semisimple. So by Lemma 2 in $[\mathrm{F}]$, the representation $\rho^{\prime}: W^{\prime} / I_{1} \rightarrow \mathrm{GL}(V)$ is semisimple. Since the image of $h^{m}$ in $W^{\prime} / I_{1}$ lies in the center, $\rho^{\prime}\left(h^{m}\right)$ is semisimple by Schur's lemma. So $\rho^{\prime}(h)$ is semisimple. This proves (i).

(ii) Keep the notations in the proof of (i). Suppose $\rho$ is semisimple. Then the restriction $\left.\rho\right|_{I_{1}}$ of $\rho$ to $I_{1}$ is semisimple since $I_{1}$ is normal in $\operatorname{Gal}(\bar{K} / K)$. But $I_{1}$ acts unipotently on $V$. So $\left.\rho\right|_{I_{1}}$ is trivial and $N=0$. Therefore $\rho(I)=\rho\left(I / I_{1}\right)$ is finite. Moreover since $N=0$, we have $\rho^{\prime}=\rho$. In the above proof of (i), if we take $h$ to be an element of degree 1 , then we have $W^{\prime}=W(\bar{K} / K)$. By assumption, $\rho: W(\bar{K} / K) \rightarrow \mathrm{GL}(V)$ is semisimple. So the representation $\rho^{\prime}: W^{\prime} / I_{1} \rightarrow \mathrm{GL}(V)$ is semisimple. Again by Schur's lemma $\rho^{\prime}\left(h^{m}\right)$ is semisimple. So $\rho(h)$ is semisimple. Therefore $\rho$ is $F$-semisimple by (i).

Now suppose $\rho$ is $F$-semisimple and $\rho(I)$ is finite. Then the restriction $\left.\rho\right|_{I}$ of $\rho$ to $I$ is semisimple. Since $I_{1}$ is normal in $I$, the restriction $\left.\rho\right|_{I_{1}}$ to $I_{1}$ is also semisimple. As above, this implies $\rho=\rho^{\prime}$. Again we take $h$ to be an element of degree 1 so that $W^{\prime}=W(\bar{K} / K)$. By assumption $\rho(h)$ is semisimple. As in the proof of (i), we can use Lemma 2 in $[\mathrm{F}]$ to deduce that $\rho$ is semisimple.

(iii) follows from the definition of $F$-semisimplicity and (i).

(iv) Let $L^{\prime}$ be a finite normal extension of $K$ contained in $\bar{K}$ and containing $L$. Put $G=\operatorname{Gal}(\bar{K} / K), H=\operatorname{Gal}(\bar{K} / L)$ and $H^{\prime}=\operatorname{Gal}\left(\bar{K} / L^{\prime}\right)$. Then $H^{\prime} \subset H \subset G$ 
and $H^{\prime}$ is normal in $G$. We have an epimorphism

$$
\begin{aligned}
\overline{\mathbf{Q}}_{l}[G] \otimes \overline{\mathbf{Q}}_{l}\left[H^{\prime}\right] & \rightarrow \overline{\mathbf{Q}}_{l}[G] \otimes \overline{\mathbf{Q}}_{l}[H] \\
g \otimes v & \mapsto g \otimes v
\end{aligned}
$$

of $\overline{\mathbf{Q}}_{l}[G]$-modules, that is, $\operatorname{Ind}_{H}^{G}(V)$ is a quotient of $\operatorname{Ind}_{H^{\prime}}^{G} \operatorname{Res}_{H}^{H^{\prime}}(V)$. So it is enough to prove $\operatorname{Ind}_{H^{\prime}}^{G} \operatorname{Res}_{H}^{H^{\prime}}(V)$ is $F$-semisimple. By (iii), $\operatorname{Res}_{H}^{H^{\prime}}(V)$ is $F$-semisimple. So replacing $L$ by $L^{\prime}$, we are reduced to proving (iv) with the additional assumption that $L / K$ is normal.

Again let $G=\operatorname{Gal}(\bar{K} / K)$ and $H=\operatorname{Gal}(\bar{K} / L)$. Now $H$ is normal in $G$. Let $g_{1} H, \ldots, g_{k} H$ be all the distinct elements in $G / H$ and let $\left\{v_{1}, \ldots, v_{l}\right\}$ be a basis for $V$. Then $g_{i} \otimes v_{j},(i=1, \ldots, k ; j=1, \ldots, l)$ form a basis for $\overline{\mathbf{Q}}_{l}[G] \otimes \overline{\mathbf{Q}}_{l}[H] V$. Let $h \in H$ be an element in the Weil group of $L$ but not in the inertia group. Since $H$ is normal in $G$, we have $h\left(g_{i} \otimes v_{j}\right)=g_{i} \otimes g_{i}^{-1} h g_{i}\left(v_{j}\right)$. But $g_{i}^{-1} h g_{i}$ acts semisimply on $V$ since $V$ is $F$-semisimple. So $h$ acts semisimply on $\overline{\mathbf{Q}}_{l}[G] \otimes \overline{\mathbf{Q}}_{l}[H] V$. Note that $h$ is also an element in the Weil group of $K$ but not in the inertia group. So by (i), $\overline{\mathbf{Q}}_{l}[G] \otimes \overline{\mathbf{Q}}_{l}[H] V$ is $F$-semisimple.

Lemma 1.2. Let $S_{0}$ be a smooth connected curve over $\mathbf{F}_{q}$ and let $\mathcal{F}_{0}$ be a lisse puncturely ı-pure $\overline{\mathbf{Q}}_{l}$-sheaf on $S_{0}$.

(i) Let $u_{0}: S_{0}^{\prime} \rightarrow S_{0}$ be a finite dominant morphism from a smooth connected curve $S_{0}^{\prime}$ to $S_{0}$, let $\bar{S}_{0}^{\prime}$ be the smooth compactification of $S_{0}^{\prime}$, and let $j: S_{0}^{\prime} \rightarrow \bar{S}_{0}^{\prime}$ be the open immersion. If $\mathcal{F}_{0}$ is locally semisimple, then for any closed point $x$ in $\bar{S}_{0}^{\prime}$, the Frobenius automorphism $F_{x}$ acts semisimply on the stalk $\left(j_{*} u_{0}^{*} \mathcal{F}_{0}\right)_{\bar{x}}$.

(ii) Conversely, let $S_{0}^{\prime}$ be a smooth connected curve and $u_{0}: S_{0}^{\prime} \rightarrow S_{0}$ a finite dominant morphism. For every closed point $x$ in $\bar{S}_{0}^{\prime}$, let $\eta_{x}$ be the generic point of the henselization of $\bar{S}_{0}^{\prime}$ at $x$ and let $\bar{\eta}_{x}$ be a geometric generic point of the strict henselization of $\bar{S}_{0}^{\prime}$ at $\bar{x}$. If for every closed point $x$ in $\bar{S}_{0}^{\prime}$, the inertia subgroup of $\operatorname{Gal}\left(\bar{\eta}_{x} / \eta_{x}\right)$ acts unipotently on $\left(u_{0}^{*} \mathcal{F}_{0}\right)_{\bar{\eta}_{x}}$, and the Frobenius automorphism $F_{x}$ acts semisimply on $\left(j_{*} u_{0}^{*} \mathcal{F}_{0}\right)_{\bar{x}}$, then $\mathcal{F}_{0}$ is locally semisimple.

Proof. (i) By Lemma 1.1 (iii), $u_{0}^{*} \mathcal{F}_{0}$ is locally semisimple. Replacing $S_{0}$ by $S_{0}^{\prime}$, we are reduced to the case $S_{0}^{\prime}=S_{0}$.

Let $I$ be the inertia group of $\operatorname{Gal}\left(\bar{\eta}_{x} / \eta_{x}\right)$. Choose an element $F \in \operatorname{Gal}\left(\bar{\eta}_{x} / \eta_{x}\right)$ lifting $F_{x} \in \operatorname{Gal}(\bar{k}(x) / k(x))$. By the definition of local semisimplicity, $F$ acts semisimply on $\mathcal{F}_{\bar{\eta}_{x}}$. In particular, it acts semisimply on $\mathcal{F}_{\bar{\eta}_{x}}^{I}$, that is, $F_{x}$ acts semisimply on $\left(j_{*} \mathcal{F}_{0}\right)_{\bar{x}}$.

(ii) The proof is similar to [D1] 1.8.4. Let $V=\left(u_{0}^{*} \mathcal{F}_{0}\right)_{\bar{\eta}_{x}}$. Since the inertia group $I$ at $x$ acts unipotently on $V$, there exists a nilpotent transformation $N: V(1) \rightarrow V$ such that any element $\sigma$ in $I$ acts on $V$ by the formula $\exp \left(t_{l}(\sigma) . N\right)$. We then have $\left(j_{*} u_{0}^{*} \mathcal{F}_{0}\right)_{\bar{x}}=\operatorname{ker}(N)$. By assumption $F_{x}$ acts semisimply on $\operatorname{ker}(N)$. So by [D1] 1.6.14, $F_{x}$ acts semisimply on $\operatorname{Gr}_{i}^{M}(V)$, where $M$ is the local monodromy filtration for $V$. Choose an element $F \in \operatorname{Gal}\left(\bar{\eta}_{x} / \eta_{x}\right)$ lifting $F_{x} \in \operatorname{Gal}(\bar{k}(x) / k(x))$. Then $F$ acts semisimply on each $\operatorname{Gr}_{i}^{M}(V)$. Let $w$ be the weight of $\mathcal{F}_{0}$. By [D1 1.8.4, the weight of $\operatorname{Gr}_{i}^{M}(V)$ is $w+i$. So $F$ acts semisimply on $V$. Hence by Lemma 1.1 (i), $u_{0}^{*} \mathcal{F}_{0}$ is locally semisimple, and by Lemma 1.1 (iii), $\mathcal{F}_{0}$ is semisimple.

Lemma 1.3. We use the same notations as in $[\mathrm{L}]$ except that we use FT to denote Fourier transformations. Let $T$ and $T^{\prime}$ be the spectra of henselian discrete valuation rings with finite residue field $\mathbf{F}_{q}$, let $\eta$ and $\eta^{\prime}$ be their generic points, and let $\rho$ : 
$\operatorname{Gal}(\bar{\eta} / \eta) \rightarrow \mathrm{GL}(\mathrm{V})$ be a $\overline{\mathbf{Q}}_{l}$-representation. Denote all the three local Fourier transformations $F T^{\left(0, \infty^{\prime}\right)}(V), F T^{\left(\infty, 0^{\prime}\right)}(V)$ and $F T^{\left(\infty, \infty^{\prime}\right)}(V)$ by $F T(V)$. If $V$ is $F$-semisimple, and if the weights of $F T(V)$ are integers (cf. [D1] 1.7.4), then $F T(V)$ is F-semisimple.

Proof. First we prove that if $V$ has finite local monodromy, that is, if the image of the inertia group $\rho(I)$ is finite, then $F T(V)$ has finite local monodromy. Note that $\widetilde{T}=T \otimes_{\mathbf{F}_{q}} \mathbf{F}$ and $\widetilde{T}^{\prime}=T^{\prime} \otimes \mathbf{F}_{q} \mathbf{F}$ are the strict henselizations of $T$ and $T^{\prime}$, respectively. Denote the three local Fourier transformations on $\widetilde{T}$ and $\widetilde{T}^{\prime}$ by $\widetilde{F T}$. Since local Fourier transformations commute with base extension $\mathbf{F}_{q} \rightarrow \mathbf{F}$, the restriction of the representation $F T(V)$ to the inertia group $I^{\prime}$ of $\operatorname{Gal}\left(\bar{\eta}^{\prime} / \eta^{\prime}\right)$ is $\widetilde{F T}(V)$. If $V$ has finite local monodromy, then its restriction to the inertia group $I$ is a semisimple representation. So $\widetilde{F T}(V)$ is semisimple, that is, the restriction of $F T(V)$ to the inertia group $I^{\prime}$ is a semisimple representation. (Recall that by the inversion formula for local Fourier transformations ([L] 2.4.3), FT transforms a semisimple representation into a semisimple representation.) But the restriction of $F T(V)$ to $I^{\prime}$ is quasi-unipotent. So the local monodromy of $F T(V)$ must be finite.

By the inversion formula for local Fourier transformations, if the local monodromy of $F T(V)$ is finite, then the local monodromy of $V$ is also finite.

Now assume $V$ is $F$-semisimple and let $M^{\prime}$ be the weight filtration of $F T(V)$. Since local Fourier transformations are exact and faithful ([L 2.4.3), we have a filtration $M=F T^{-1}\left(M^{\prime}\right)$ on $V$ and $F T\left(\operatorname{Gr}_{i}^{M}(V)\right)=\operatorname{Gr}_{i}^{M^{\prime}} F T(V)$. By [D1] 1.7.5, we have $N M_{i}^{\prime}(1) \subset M_{i-2}^{\prime}$, where $N$ is defined as in the proof of Lemma 1.1 (i). Hence $N$ acts trivially on $\operatorname{Gr}_{i}^{M^{\prime}} F T(V)$. So the local monodromy of $F T\left(\mathrm{Gr}_{i}^{M}(V)\right)=\mathrm{Gr}_{i}^{M^{\prime}} F T(V)$ is finite. By what we proved above, the local monodromy of $\operatorname{Gr}_{i}^{M}(V)$ is also finite. Since $V$ is $F$-semisimple, $\operatorname{Gr}_{i}^{M}(V)$ is also $F$ semisimple. By Lemma 1.1 (ii), $\operatorname{Gr}_{i}^{M}(V)$ is semisimple. So $F T\left(\operatorname{Gr}_{i}^{M}(V)\right)$ is semisimple, that is, $\mathrm{Gr}_{i}^{M^{\prime}} F T(V)$ is semisimple. By Lemma 1.1 (ii), every element in the Weil group $W\left(\bar{\eta}^{\prime} / \eta^{\prime}\right)$ but not in the inertia group $I^{\prime}$ acts semisimply on $\mathrm{Gr}_{i}^{M^{\prime}} F T(V)$. But the weights of $\mathrm{Gr}_{i}^{M^{\prime}} F T(V)$ are distinct for different $i$ (cf. [D1 1.7.5.) So every element in $W\left(\bar{\eta}^{\prime} / \eta^{\prime}\right)-I^{\prime}$ acts semisimply on $F T(V)$ and $F T(V)$ is $F$-semisimple.

We also need the following lemma of Katz ([K] Lemma 16).

Lemma 1.4. Let $S_{0}$ be a smooth geometrically connected curve over $\mathbf{F}_{q}$. Then there exists a nonempty open subset $U_{0}$ of $S_{0}$ such that there exists a finite étale morphism $f_{0}: U_{0} \rightarrow \mathbf{A}^{1}$, where $\mathbf{A}^{1}$ is the affine line over $\mathbf{F}_{q}$.

Now we are ready to prove Theorem 2 . We may assume the weight of $\mathcal{F}_{0}$ is an integer by twisiting. By Theorem 1 , the locally semisimple sheaf $\mathcal{F}_{0}$ is semisimple, that is, $\mathcal{F}_{\bar{x}}$ is a semisimple representation of $\pi_{1}\left(S_{0}, \bar{x}\right)$, where $x$ is any closed point of $S_{0}$. Since $\pi_{1}(S, \bar{x})$ is a normal subgroup of $\pi_{1}\left(S_{0}, \bar{x}\right), \mathcal{F}_{\bar{x}}^{\pi_{1}(S, \bar{x})}$ is a semisimple representation of $\pi_{1}\left(S_{0}, \bar{x}\right) / \pi_{1}(S, \bar{x}) \cong \operatorname{Gal}\left(\mathbf{F} / \mathbf{F}_{q}\right)$. So $F$ acts semisimply on $H^{0}\left(\bar{S}, j_{*} \mathcal{F}\right)=\mathcal{F}_{\bar{x}}^{\pi_{1}(S, \bar{x})}$. By [SGA4 XVIII 3.2.5 and SGA4 $\frac{1}{2}$. Dualité, 1.3, we have a perfect paring between $H^{2}\left(\bar{S}, j_{*} \mathcal{F}\right)$ and $H^{0}\left(\bar{S}, j_{*} \check{\mathcal{F}}(1)\right)$. So $F$ acts semisimply on $H^{2}\left(\bar{S}, j_{*} \mathcal{F}\right)$. It remains to show $F$ acts semisimply on $H^{1}(\bar{S}, \mathcal{F})$.

By Lemma 1.4, we may choose an open subset $U_{0}$ so that there exists a finite étale morphism $f_{0}: U_{0} \rightarrow \mathbf{A}^{1}$. Obviously $\left.\mathcal{F}_{0}\right|_{U_{0}}$ is locally semisimple and $\iota$-pure. Since $\mathcal{F}_{0}$ is lisse on $S_{0}$, we have $\mathcal{F}_{0}=l_{*}\left(\left.\mathcal{F}_{0}\right|_{U_{0}}\right)$, where $l: U_{0} \rightarrow S_{0}$ is the open 
immersion. So replacing $S_{0}$ by $U_{0}$, it is enough to prove Theorem 2 with the additional assumption that there is a finite étale morphism $f_{0}: S_{0} \rightarrow \mathbf{A}^{1}$. Let $\bar{f}_{0}: \bar{S}_{0} \rightarrow \mathbf{P}^{1}$ be the extension of $f_{0}$. It is a finite morphism.

We have

$$
H^{1}\left(\bar{S}, j_{*} \mathcal{F}\right)=H^{1}\left(\mathbf{P}^{1}, \bar{f}_{*} j_{*} \mathcal{F}\right)=H^{1}\left(\mathbf{P}^{1}, j_{*} f_{*} \mathcal{F}\right),
$$

where we denote the open immersion $\mathbf{A}^{1} \rightarrow \mathbf{P}^{1}$ also by $j$. So we need to show $F$ acts semisimply on $H^{1}\left(\mathbf{P}^{1}, j_{*} f_{*} \mathcal{F}\right)$. Since $f_{0}$ is finite and étale, $f_{0 *} \mathcal{F}_{0}$ is lisse. By Lemma 1.1 (iv), the sheaf $f_{0 *} \mathcal{F}_{0}$ is locally semisimple, and by [D1] 1.2 .5 (i), it is $\iota$-pure. So replacing $S_{0}$ by $\mathbf{A}^{1}$ and $\mathcal{F}_{0}$ by $f_{0 *} \mathcal{F}_{0}$, we are reduced to proving Theorem 2 with the additional assumption that $S_{0}=\mathbf{A}^{1}$.

Let $\mathbf{G}_{\mathbf{m}}=\mathbf{A}^{1}-\{0\}$. As we have shown above, it is enough to prove $F$ acts semisimply on $H^{1}\left(\mathbf{P}^{1}, k_{*}\left(\left.\mathcal{F}\right|_{\mathbf{G}_{\mathbf{m}}}\right)\right)$, where $k: \mathbf{G}_{\mathbf{m}} \rightarrow \mathbf{P}^{1}$ is the open immersion. Note that $\left.\mathcal{F}_{0}\right|_{\mathbf{G}_{\mathbf{m}}}$ is unramified at 0 . Making a coordinate change $x \mapsto 1 / x$, we are reduced to proving Theorem 2 with the additional assumption that $S_{0}=\mathbf{G}_{\mathbf{m}}$ and $\mathcal{F}_{0}$ is unramified at $\infty$.

Let $k: \mathbf{G}_{\mathbf{m}} \rightarrow \mathbf{P}^{1}$ and $j: \mathbf{G}_{\mathbf{m}} \rightarrow \mathbf{A}^{1}$ be the open immersions. Obviously $\left.\left(k_{*} \mathcal{F}_{0}\right)\right|_{\mathbf{A}^{1}}=j_{*} \mathcal{F}_{0}$. We have an exact sequence

$$
H_{c}^{1}\left(\mathbf{A}^{1}, j_{*} \mathcal{F}\right) \rightarrow H^{1}\left(\mathbf{P}^{1}, k_{*} \mathcal{F}\right) \rightarrow 0
$$

since $H^{1}\left(\mathbf{P}^{1}-\mathbf{A}^{1},\left.\left(k_{*} \mathcal{F}\right)\right|_{\mathbf{P}^{1}-\mathbf{A}^{1}}\right)=0$. To prove $F$ acts semisimply on $H^{1}\left(\mathbf{P}^{1}, k_{*} \mathcal{F}\right)$, it suffices to prove $F$ acts semisimply on $H_{c}^{1}\left(\mathbf{A}^{1}, j_{*} \mathcal{F}\right)$.

Let's summarize what we get. We have a sheaf $\mathcal{F}_{0}$ on $\mathbf{G}_{\mathbf{m}}$. It is lisse, locally semisimple, $\iota$-pure with integer weight, and unramified at $\infty$. We need to show $F$ acts semisimply on $H_{c}^{1}\left(\mathbf{A}^{1}, j_{*} \mathcal{F}\right)$. We prove this using $l$-adic Fourier transformations and local Fourier transformations.

We use the same notations as in [L] except that we use FT to denote the Fourier transformation. Since $\mathcal{F}_{0}$ is necessarily semisimple, we may assume $\mathcal{F}_{0}$ is irreducible. By [L] 1.4.2.1 and 2.3.1.3 (i), we have

$$
F T\left(j_{*} \mathcal{F}_{0}[1]\right)=j_{*}^{\prime} \mathcal{F}_{0}^{\prime}[1],
$$

where $j^{\prime}: \mathbf{A}^{1 \prime}-\left\{0^{\prime}\right\} \rightarrow \mathbf{A}^{1 \prime}$ is the open immersion, $\mathbf{A}^{1 \prime}$ is the dual of $\mathbf{A}^{1}$ and $0^{\prime}$ is the origin of $\mathbf{A}^{1 \prime}$, and $\mathcal{F}_{0}^{\prime}$ is some irreducible lisse $\overline{\mathbf{Q}}_{l^{-}}$sheaf on $\mathbf{A}^{1 \prime}-\left\{0^{\prime}\right\}$. We have a distinguished triangle in the derived category of $\overline{\mathbf{Q}}_{l}$-sheaves

$$
j_{*} \mathcal{F}_{0} / j_{!} \mathcal{F}_{0} \rightarrow j_{!} \mathcal{F}_{0}[1] \rightarrow j_{*} \mathcal{F}_{0}[1] \rightarrow
$$

It induces the distinguished triangle

$$
F T\left(j_{*} \mathcal{F}_{0} / j_{!} \mathcal{F}_{0}\right) \rightarrow F T\left(j_{!} \mathcal{F}_{0}[1]\right) \rightarrow F T\left(j_{*} \mathcal{F}_{0}[1]\right) \rightarrow
$$

But $F T\left(j_{*} \mathcal{F}_{0}[1]\right)=j_{*}^{\prime} \mathcal{F}_{0}^{\prime}[1]$, and by [L] 1.2.2.2 and 1.2.2.1, we have $F T\left(j_{*} \mathcal{F}_{0} / j_{!} \mathcal{F}_{0}\right)$ $=V[1]$, where $V$ is the inverse image of the sheaf $\left.\left(j_{*} \mathcal{F}_{0}\right)\right|_{\{0\}}$ on $\{0\} \cong \operatorname{spec} \mathbf{F}_{q}$ under the structure morphism $\mathbf{A}^{1 \prime} \rightarrow \operatorname{spec} \mathbf{F}_{q}$. So we have a distinguished triangle

$$
V[1] \rightarrow F T\left(j_{!} \mathcal{F}_{0}[1]\right) \rightarrow j_{*}^{\prime} \mathcal{F}_{0}^{\prime}[1] \rightarrow .
$$

Taking the cohomology groups of the complexes in this last triangle, we get the exact sequence

$$
0 \rightarrow V \rightarrow \mathcal{H}^{-1}\left(F T\left(j_{!} \mathcal{F}_{0}[1]\right)\right) \rightarrow j_{*}^{\prime} \mathcal{F}_{0}^{\prime} \rightarrow 0
$$

and $\mathcal{H}^{i}\left(F T\left(j_{!} \mathcal{F}_{0}[1]\right)\right)=0$ for $i \neq-1$. 
By [L] 2.3.3.1 (iii), we have

$$
\left(\mathcal{H}^{-1}\left(F T\left(j ! \mathcal{F}_{0}[1]\right)\right)\right)_{\bar{\eta}_{\infty^{\prime}}}=F T^{\left(0, \infty^{\prime}\right)}\left(\mathcal{F}_{\bar{\eta}_{0}}\right) \oplus F T^{\left(\infty, \infty^{\prime}\right)}\left(\mathcal{F}_{\bar{\eta}_{\infty}}\right)
$$

By our assumption, $\mathcal{F}_{0}$ is unramified at $\infty$, so $F T^{\left(\infty, \infty^{\prime}\right)}\left(\mathcal{F}_{\bar{\eta}_{\infty}}\right)=0$. Therefore,

$$
\left(\mathcal{H}^{-1}\left(F T\left(j_{!} \mathcal{F}_{0}[1]\right)\right)\right)_{\bar{\eta}_{\infty^{\prime}}}=F T^{\left(0, \infty^{\prime}\right)}\left(\mathcal{F}_{\bar{\eta}_{0}}\right) .
$$

So the above exact sequence induces the following one:

$$
0 \rightarrow V \rightarrow F T^{\left(0, \infty^{\prime}\right)}\left(\mathcal{F}_{\bar{\eta}_{0}}\right) \rightarrow \mathcal{F}_{\bar{\eta}_{\infty^{\prime}}}^{\prime} \rightarrow 0
$$

Let $w$ be the weight of $\mathcal{F}_{0}$. By assumption, it is an integer. By $[\mathrm{L}] 4.4, \mathcal{F}_{0}^{\prime}$ is $\iota$-pure of (integer) weight $w+1$. By [D1] 1.8.4, the weights of $V=\left.\left(j_{*} \mathcal{F}\right)\right|_{\{0\}}$ and of $\mathcal{F}_{\bar{\eta}_{\infty}}^{\prime}$ are integers. So by the last exact sequence, the weights of $F T^{\left(0, \infty^{\prime}\right)}\left(\mathcal{F}_{\bar{\eta}_{0}}\right)$ are integers. By assumption $\mathcal{F}_{\bar{\eta}_{0}}$ is $F$-semisimple. So by Lemma 1.3, $F T^{\left(0, \infty^{\prime}\right)}\left(\mathcal{F}_{\bar{\eta}_{0}}\right)$ is $F$-semisimple. By the last exact sequence, $\mathcal{F}_{\bar{\eta}_{\infty}}^{\prime}$ is $F$-semisimple. By the main conjecture, $\mathcal{F}_{\bar{\eta}_{0}}^{\prime}$ is $F$-semisimple. By Lemma $1.2(\mathrm{i}), F$ acts semisimply on $\left(j_{*}^{\prime} \mathcal{F}^{\prime}\right)_{\overline{0}}$. But $\left(j_{*}^{\prime} \mathcal{F}^{\prime}\right)_{\overline{0}}=\left(F T\left(j_{*} \mathcal{F}\right)\right)_{\overline{0}}=H_{c}^{1}\left(\mathbf{A}^{\mathbf{1}}, j_{*} \mathcal{F}\right)$. So $F$ acts semisimply on $H_{c}^{1}\left(\mathbf{A}^{\mathbf{1}}, j_{*} \mathcal{F}\right)$. This proves our assertion.

\section{Proof of Theorem 3}

We prove Theorem 3 by induction on the dimension of the scheme. Assume the semisimplicity conjecture holds for schemes with dimension less than or equal to $n$. Let $X_{0}$ be a smooth projective scheme over $\mathbf{F}_{q}$ of dimension $n+1$. Embed $X$ into a projective space $\mathbf{P}$ and sweep $X$ by a Lefschetz pencil of hyperplanes $\left\{H_{t}\right\}_{t \in D}$, where $D$ is a projective line in the dual $\check{\mathbf{P}}$ of $\mathbf{P}$. Let $\widetilde{X}=\left\{(x, t) \in X \times D \mid x \in H_{t}\right\}$ be the blowing-up of $X$ along the intersection of $X$ with the axis of the pencil, let $f: \widetilde{X} \rightarrow D$ be the canonical projection, and let $S \subset D$ be the set of critical values of $f$. Making a base extension if necessary, we may assume the pencil and $f$ are defined over $\mathbf{F}_{q}$ and $S$ consists of $\mathbf{F}_{q}$-rational points. Let $f_{0}: \widetilde{X}_{0} \rightarrow D_{0}$ be the canonical projection and let $i_{0}: S \rightarrow D_{0}$ and $j_{0}: D_{0}-S \rightarrow D_{0}$ be the immersions. These morphisms are all defined over $\mathbf{F}_{q}$.

We know $H^{i}\left(X, \overline{\mathbf{Q}}_{l}\right)$ is a subspace of $H^{i}\left(\widetilde{X}, \overline{\mathbf{Q}}_{l}\right)$ as $\operatorname{Gal}\left(\mathbf{F} / \mathbf{F}_{q}\right)$-modules ([SGA7] XVIII 4.2). So to prove the geometric Frobenius correspondence $F$ acts semisimply on $H^{i}\left(X, \overline{\mathbf{Q}}_{l}\right)$, it suffices to show $F$ acts semisimply on $H^{i}\left(\widetilde{X}, \overline{\mathbf{Q}}_{l}\right)$.

The following result follows directly from the Picard-Lefschetz formula ([SGA7] XV 3.4):

Proposition 2.1. Notation as above.

(i) If the vanishing cycles are not zero, then for $i \neq n, R^{i} f_{0 *} \overline{\mathbf{Q}}_{l}$ is a lisse sheaf on $D_{0}, j_{0}^{*} R^{n} f_{0 *} \overline{\mathbf{Q}}_{l}$ is a lisse sheaf on $D_{0}-S$ and $R^{n} f_{0 *} \overline{\mathbf{Q}}_{l}=j_{0 *} j_{0}^{*} R^{n} f_{0 *} \overline{\mathbf{Q}}_{l}$.

(ii) If the vanishing cycles are zero, then $n$ is necessarily odd, and for $i \neq n+1$, $R^{i} f_{0 *} \overline{\mathbf{Q}}_{l}$ and $j_{0 *} j_{0}^{*} R^{n+1} f_{0 *} \overline{\mathbf{Q}}_{l}$ are lisse sheaves on $D_{0}$ and we have an exact sequence

$$
0 \rightarrow i_{0 *} \overline{\mathbf{Q}}_{l}(m-n) \rightarrow R^{n+1} f_{0 *} \overline{\mathbf{Q}}_{l} \rightarrow j_{0 *} j_{0}^{*} R^{n+1} f_{0 *} \overline{\mathbf{Q}}_{l} \rightarrow 0,
$$

where $m$ is defined by $n=2 m+1$.

(Note that since vanishing cycles are conjugate under the action of $\pi_{1}(D-S)$, if one vanishing cycle is zero, then all vanishing cycles are zero.) 
To go on, we have to use the language of perverse sheaves. We use the same notation as in [BBD]. For example, ${ }^{p} R^{i} f_{0 *} \overline{\mathbf{Q}}_{l}$ is the $i$-th perverse cohomology sheaf of $R f_{0 *} \overline{\mathbf{Q}}_{l}$ in the derived category $D_{c}^{b}\left(D_{0}, \overline{\mathbf{Q}}_{l}\right)$ of $\overline{\mathbf{Q}}_{l}$-sheaves on $D_{0}$.

Lemma 2.2. (i) If the vanishing cycles are not zero, then

$$
{ }^{p} R^{i} f_{0 *} \overline{\mathbf{Q}}_{l}=R^{i-1} f_{0 *} \overline{\mathbf{Q}}_{l}[1]
$$

for all $i$.

(ii) If the vanishing cycles are zero, then $n$ is necessarily odd, and for $i \neq n+$ $1, n+2$, we have

$$
{ }^{p} R^{i} f_{0 *} \overline{\mathbf{Q}}_{l}=R^{i-1} f_{0 *} \overline{\mathbf{Q}}_{l}[1],
$$

and

$$
\begin{aligned}
{ }^{p} R^{n+1} f_{0 *} \overline{\mathbf{Q}}_{l} & =R^{n} f_{0 *} \overline{\mathbf{Q}}_{l}[1] \oplus i_{0 *} \overline{\mathbf{Q}}_{l}(m-n), \\
{ }^{p} R^{n+2} f_{0 *} \overline{\mathbf{Q}}_{l} & =j_{0 *} j_{0}^{*} R^{n+1} f_{0 *} \overline{\mathbf{Q}}_{l}[1],
\end{aligned}
$$

where $m$ is defined by $n=2 m+1$.

Proof. We give the proof of (ii). The proof of (i) is similar and is left to the reader.

Denote by $\tau_{\geq i} K$ the truncation of a complex $K$ with respect to the standard $t$-structure on $D_{c}^{b}\left(D_{0}, \overline{\mathbf{Q}}_{l}\right)$. Since $R^{i} f_{0 *} \overline{\mathbf{Q}}_{l}$ vanishes for $i \notin[0,2 n]$, we have the following distinguished triangles:

$$
\begin{array}{ccccccc}
R^{2 n-1} f_{0 *} \overline{\mathbf{Q}}_{l}[-(2 n-1)] & \rightarrow & \tau_{\geq 2 n-1} R f_{0 *} \overline{\mathbf{Q}}_{l} & \rightarrow & R^{2 n} f_{0 *} \overline{\mathbf{Q}}_{l}[-2 n] & \rightarrow, \\
R^{2 n-2} f_{0 *} \overline{\mathbf{Q}}_{l}[-(2 n-2)] & \rightarrow & \tau_{\geq 2 n-2} R f_{0 *} \overline{\mathbf{Q}}_{l} & \rightarrow & \tau_{\geq 2 n-1} R f_{0 *} \overline{\mathbf{Q}}_{l} & \rightarrow, \\
\vdots & & & & & \\
R^{n+1} f_{0 *} \overline{\mathbf{Q}}_{l}[-(n+1)] & \rightarrow & \tau_{\geq n+1} R f_{0 *} \overline{\mathbf{Q}}_{l} & \rightarrow & \tau_{\geq n+2} R f_{0 *} \overline{\mathbf{Q}}_{l} & \rightarrow, \\
R^{n} f_{0 *} \overline{\mathbf{Q}}_{l}[-n] & \rightarrow & \tau_{\geq n} R f_{0 *} \overline{\mathbf{Q}}_{l} & \rightarrow & \tau_{\geq n+1} R f_{0 *} \overline{\mathbf{Q}}_{l} & \rightarrow, \\
\vdots & & & & & \\
R^{1} f_{0 *} \overline{\mathbf{Q}}_{l}[-1] & \rightarrow & \tau_{\geq 1} R f_{0 *} \overline{\mathbf{Q}}_{l} & \rightarrow & \tau_{\geq 2} R f_{0 *} \overline{\mathbf{Q}}_{l} & \rightarrow, \\
R^{0} f_{0 *} \overline{\mathbf{Q}}_{l} & \rightarrow & R f_{0 *} \overline{\mathbf{Q}}_{l} & \rightarrow & \tau_{\geq 1} R f_{0 *} \overline{\mathbf{Q}}_{l} & \rightarrow .
\end{array}
$$

By Proposition 2.1 (ii), for $i \neq n+1, R^{i} f_{0 *} \overline{\mathbf{Q}}_{l}[1]$ is perverse, and $R^{n+1} f_{0 *} \overline{\mathbf{Q}}_{l}$ lies in ${ }^{p} D_{c}^{[0,1]}\left(D_{0}, \overline{\mathbf{Q}}_{l}\right)$. The above list of distinguished triangles then shows that

$$
\begin{aligned}
& R^{2 n} f_{0 *} \overline{\mathbf{Q}}_{l}[-2 n] \quad \in{ }^{p} D^{\geq 2 n+1} \cap{ }^{p} D^{\leq 2 n+1}, \\
& R^{2 n-1} f_{0 *} \overline{\mathbf{Q}}_{l}[-(2 n-1)] \in{ }^{p} D^{\geq 2 n} \cap{ }^{p} D^{\leq 2 n}, \quad \tau_{\geq 2 n-1} R f_{0 *} \overline{\mathbf{Q}}_{l} \in{ }^{p} D^{[2 n, 2 n+1]}, \\
& R^{2 n-2} f_{0 *} \overline{\mathbf{Q}}_{l}[-(2 n-2)] \in{ }^{p} D^{\geq 2 n-1} \cap{ }^{p} D^{\leq 2 n-1}, \tau_{\geq 2 n-2} R f_{0 *} \overline{\mathbf{Q}}_{l} \in{ }^{p} D^{[2 n-1,2 n+1]} \text {, } \\
& R^{n+2} f_{0 *} \overline{\mathbf{Q}}_{l}[-(n+2)] \in{ }^{p} D^{\geq n+3} \cap{ }^{p} D^{\leq n+3}, \quad \tau_{\geq n+2} R f_{0 *} \overline{\mathbf{Q}}_{l} \in{ }^{p} D^{[n+3,2 n+1]}, \\
& R^{n+1} f_{0 *} \overline{\mathbf{Q}}_{l}[-(n+1)] \in{ }^{p} D^{[n+1, n+2]}, \quad \tau_{\geq n+1} R f_{0 *} \overline{\mathbf{Q}}_{l} \in{ }^{p} D^{[n+1,2 n+1]}, \\
& R^{n} f_{0 *} \overline{\mathbf{Q}}_{l}[-n] \quad \in{ }^{p} D^{\geq n+1} \cap{ }^{p} D^{\leq n+1}, \quad \tau_{\geq n} R f_{0 *} \overline{\mathbf{Q}}_{l} \in{ }^{p} D^{[n+1,2 n+1]}, \\
& \begin{array}{cccccc}
\vdots & & & & & \\
R^{1} f_{0 *} \overline{\mathbf{Q}}_{l}[-1] & \in & { }^{p} D^{\geq 2} \cap^{p} D^{\leq 2}, & \tau_{\geq 1} R f_{0 *} \overline{\mathbf{Q}}_{l} & \in & { }^{p} D^{[2,2 n+1]}, \\
R^{0} f_{0 *} \overline{\mathbf{Q}}_{l} & \in & { }^{p} D^{\geq 1} \cap^{p} D^{\leq 1}, & R f_{0 *} \mathbf{Q}_{l} & \in & { }^{p} D^{[1,2 n+1]} .
\end{array}
\end{aligned}
$$

These facts and the list of triangles show that ${ }^{p} R^{i} f_{0 *} \overline{\mathbf{Q}}_{l}=R^{i-1} f_{0 *} \overline{\mathbf{Q}}_{l}[1]$ for $i \leq n$ and $\tau_{\geq n} R f_{0 *} \overline{\mathbf{Q}}_{l}={ }^{p} \tau_{\geq n+1} R f_{0 *} \overline{\mathbf{Q}}_{l}$, where ${ }^{p} \tau_{\geq}$is the truncation with repect to the perverse $t$-structure. 
From the long exact sequence of perverse cohomology sheaves for the distinguished triangle

$$
R^{n} f_{0 *} \overline{\mathbf{Q}}_{l}[-n] \rightarrow \tau_{\geq n} R f_{0 *} \overline{\mathbf{Q}}_{l} \rightarrow \tau_{\geq n+1} R f_{0 *} \overline{\mathbf{Q}}_{l} \rightarrow,
$$

we get the following short exact sequence of perverse sheaves:

$$
0 \rightarrow R^{n} f_{0 *} \overline{\mathbf{Q}}_{l}[1] \rightarrow{ }^{p} R^{n+1} f_{0 *} \overline{\mathbf{Q}}_{l} \rightarrow{ }^{p} \mathcal{H}^{n+1} \tau_{\geq n+1} R f_{0 *} \overline{\mathbf{Q}}_{l} \rightarrow 0,
$$

where ${ }^{p} \mathcal{H}^{i}$ denote the $i$-th pervese cohomology sheaf of a complex. From the long exact sequence of perverse cohomology sheaves for the distinguished triangle

$$
R^{n+1} f_{0 *} \overline{\mathbf{Q}}_{l}[-(n+1)] \rightarrow \tau_{\geq n+1} R f_{0 *} \overline{\mathbf{Q}}_{l} \rightarrow \tau_{\geq n+2} R f_{0 *} \overline{\mathbf{Q}}_{l} \rightarrow,
$$

we get the following exact sequence of perverse sheaves:

$$
0 \rightarrow{ }^{p} \mathcal{H}^{n+1} R^{n+1} f_{0 *} \overline{\mathbf{Q}}_{l}[-(n+1)] \rightarrow{ }^{p} \mathcal{H}^{n+1} \tau_{\geq n+1} R f_{0 *} \overline{\mathbf{Q}}_{l} \rightarrow 0 .
$$

By the short exact sequence in Proposition 2.1 (ii), we have

$$
{ }^{p} \mathcal{H}^{n+1} R^{n+1} f_{0 *} \overline{\mathbf{Q}}_{l}[-(n+1)]=i_{0 *} \overline{\mathbf{Q}}_{l}(m-n) .
$$

So we have the following short exact sequence of perverse sheaves:

$$
0 \rightarrow R^{n} f_{0 *} \overline{\mathbf{Q}}_{l}[1] \rightarrow{ }^{p} R^{n+1} f_{0 *} \overline{\mathbf{Q}}_{l} \rightarrow i_{0 *} \overline{\mathbf{Q}}_{l}(m-n) \rightarrow 0 .
$$

This exact sequence corresponds to an element in $\operatorname{Hom}\left(i_{0 *} \overline{\mathbf{Q}}_{l}(m-n), R^{n} f_{0 *} \overline{\mathbf{Q}}_{l}[2]\right)$. We have

$$
\begin{aligned}
\operatorname{Hom}\left(i_{0 *} \overline{\mathbf{Q}}_{l}(m-n), R^{n} f_{0 *} \overline{\mathbf{Q}}_{l}[2]\right) & =\operatorname{Hom}\left(\overline{\mathbf{Q}}_{l}(m-n), R i_{0}^{!} R^{n} f_{0 *} \overline{\mathbf{Q}}_{l}[2]\right) \\
& =\operatorname{Hom}\left(\overline{\mathbf{Q}}_{l}(m-n), i_{0}^{*} R^{n} f_{0 *} \overline{\mathbf{Q}}_{l}(-1)\right),
\end{aligned}
$$

where the second equality follows from the purity (which can be easily deduced from [SGA4] XVIII 3.2.5) and the fact that $R^{n} f_{0 *} \overline{\mathbf{Q}}_{l}$ is lisse. Note that the lisse sheaf $R^{n} f_{0 *} \overline{\mathbf{Q}}_{l}$ on $D_{0}$ is geometrically constant since $D_{0}$, being the projective line, has trivial geometric fundamental group. So $R^{n} f_{0 *} \overline{\mathbf{Q}}_{l}$ is the inverse image of a sheaf on $\operatorname{spec}_{q}$. Combining this fact with Deligne's theorem applied to the fiber of $R^{n} f_{0 *} \overline{\mathbf{Q}}_{l}$ at points in $D_{0}-S$, we see that $R^{n} f_{0 *} \overline{\mathbf{Q}}_{l}$ is puncturely pure of weight $n$. So $i_{0}^{*} R^{n} f_{0 *} \overline{\mathbf{Q}}_{l}(-1)$ is puncturely pure of weight $n+2$. But $\overline{\mathbf{Q}}_{l}(m-n)$ is puncturely pure of weight $2(n-m)=n+1$. So $\operatorname{Hom}\left(\overline{\mathbf{Q}}_{l}(m-n), i_{0}^{*} R^{n} f_{0 *} \overline{\mathbf{Q}}_{l}(-1)\right)=0$ and hence $\operatorname{Hom}\left(i_{0 *} \overline{\mathbf{Q}}_{l}(m-n), R^{n} f_{0 *} \overline{\mathbf{Q}}_{l}[2]\right)=0$. Therefore the exact sequence

$$
0 \rightarrow R^{n} f_{0 *} \overline{\mathbf{Q}}_{l}[1] \rightarrow{ }^{p} R^{n+1} f_{0 *} \overline{\mathbf{Q}}_{l} \rightarrow i_{0 *} \overline{\mathbf{Q}}_{l}(m-n) \rightarrow 0
$$

splits and hence ${ }^{p} R^{n+1} f_{0 *} \overline{\mathbf{Q}}_{l}=R^{n} f_{0 *} \overline{\mathbf{Q}}_{l}[1] \oplus i_{0 *} \overline{\mathbf{Q}}_{l}(m-n)$.

From the long exact sequence of perverse cohomology sheaves associated to the distinguished triangle

$$
R^{n+1} f_{0 *} \overline{\mathbf{Q}}_{l}[-(n+1)] \rightarrow \tau_{\geq n+1} R f_{0 *} \overline{\mathbf{Q}}_{l} \rightarrow \tau_{\geq n+2} R f_{0 *} \overline{\mathbf{Q}}_{l} \rightarrow
$$

and the fact that $\tau_{\geq n+2} R f_{0 *} \overline{\mathbf{Q}}_{l} \in{ }^{p} D^{[n+3,2 n+1]}$, we get

$$
{ }^{p} \mathcal{H}^{n+2} R^{n+1} f_{0 *} \overline{\mathbf{Q}}_{l}[-(n+1)]={ }^{p} \mathcal{H}^{n+2} \tau_{\geq n+1} R f_{0 *} \overline{\mathbf{Q}}_{l} .
$$

From the long exact sequence of perverse cohomology sheaves associated to the distinguished triangle

$$
R^{n} f_{0 *} \overline{\mathbf{Q}}_{l}[-n] \rightarrow \tau_{\geq n} R f_{0 *} \overline{\mathbf{Q}}_{l} \rightarrow \tau_{\geq n+1} R f_{0 *} \overline{\mathbf{Q}}_{l} \rightarrow
$$

and the fact that $R^{n} f_{0 *} \overline{\mathbf{Q}}_{l}[-n] \in{ }^{p} D^{\geq n+1} \cap{ }^{p} D^{\leq n+1}$, we get

$$
{ }^{p} \mathcal{H}^{n+2} \tau_{\geq n+1} R f_{0 *} \overline{\mathbf{Q}}_{l}={ }^{p} \mathcal{H}^{n+2} \tau_{\geq n} R f_{0 *} \overline{\mathbf{Q}}_{l} .
$$


Similarly,

$$
\begin{aligned}
{ }^{p} \mathcal{H}^{n+2} \tau_{\geq n} R f_{0 *} \overline{\mathbf{Q}}_{l} & ={ }^{p} \mathcal{H}^{n+2} \tau_{\geq n-1} R f_{0 *} \overline{\mathbf{Q}}_{l}=\cdots={ }^{p} \mathcal{H}^{n+2} \tau_{\geq 1} R f_{0 *} \overline{\mathbf{Q}}_{l} \\
& ={ }^{p} \mathcal{H}^{n+2} R f_{0 *} \overline{\mathbf{Q}}_{l} .
\end{aligned}
$$

So

$$
{ }^{p} R^{n+2} f_{0 *} \overline{\mathbf{Q}}_{l}={ }^{p} \mathcal{H}^{n+2} R f_{0 *} \overline{\mathbf{Q}}_{l}={ }^{p} \mathcal{H}^{n+2} R^{n+1} f_{0 *} \overline{\mathbf{Q}}_{l}[-(n+1)] .
$$

By the exact sequence in Proposition 2.1 (ii), we have

$$
{ }^{p} \mathcal{H}^{n+2} R^{n+1} f_{0 *} \overline{\mathbf{Q}}_{l}[-(n+1)]=j_{0 *} j_{0}^{*} R^{n+1} f_{0 *} \overline{\mathbf{Q}}_{l}[1]
$$

So

$$
{ }^{p} R^{n+2} f_{0 *} \overline{\mathbf{Q}}_{l}=j_{0 *} j_{0}^{*} R^{n+1} f_{0 *} \overline{\mathbf{Q}}_{l}[1] .
$$

Using a similar method, one can show that ${ }^{p} R^{i} f_{0 *} \overline{\mathbf{Q}}_{l}=R^{i-1} f_{0 *} \overline{\mathbf{Q}}_{l}[1]$ for $i \geq n+3$.

Lemma 2.3. Under the induction hypothesis,

(i) if the vanishing cycles are not zero, then for $i \neq n, R^{i} f_{0 *} \overline{\mathbf{Q}}_{l}$ is a lisse locally semisimple sheaf on $D_{0}$ and $j_{0}^{*} R^{n} f_{0 *} \overline{\mathbf{Q}}_{l}$ is a lisse locally semisimple sheaf on $D_{0}-S$;

(ii) if the vanishing cycles are zero, then for $i \neq n+1, R^{i} f_{0 *} \overline{\mathbf{Q}}_{l}$ is a lisse locally semisimple sheaf on $D_{0}$ and $j_{0}^{*} R^{n+1} f_{0 *} \overline{\mathbf{Q}}_{l}$ is a lisse locally semisimple sheaf on $D_{0}-S$.

Proof. (i) By Proposition 2.1 (i), for $i \neq n, R^{i} f_{0 *} \overline{\mathbf{Q}}_{l}$ is a lisse sheaf on $D_{0}$. Since the geometric fundamental group of $D_{0}$ is trivial, $R^{i} f_{0 *} \overline{\mathbf{Q}}_{l}$ is geometrically constant and hence is the inverse image of a sheaf on $\operatorname{spec} \mathbf{F}_{q}$. So to prove $R^{i} f_{0 *} \overline{\mathbf{Q}}_{l}$ is locally semisimple, it suffices to find one closed point $s$ in $D_{0}$ such that $F_{s}$ acts semisimply on $\left(R^{i} f_{0 *} \overline{\mathbf{Q}}_{l}\right)_{\bar{s}}=H^{i}\left(\widetilde{X}_{\bar{s}}, \overline{\mathbf{Q}}_{l}\right)$. We can take $s$ to be a point in $D_{0}-S$ and apply the induction hypothesis.

Next we prove $j_{0}^{*} R^{n} f_{0 *} \overline{\mathbf{Q}}_{l}$ is locally semisimple. Let $s$ be a closed point of $D_{0}$. If $s \in D_{0}-S$, then $F_{s}$ acts semisimply on $\left(j_{0}^{*} R^{n} f_{0 *} \overline{\mathbf{Q}}_{l}\right)_{\bar{s}}=H^{n}\left(\widetilde{X}_{\bar{s}}, \overline{\mathbf{Q}}_{l}\right)$ by the induction hypothesis. Assume $s \in S$. Let $\eta_{s}$ be the generic point of the henselization of $\bar{D}_{0}$ at $s$, and let $\bar{\eta}_{s}$ be a geometric generic point of the strict henselization of $\bar{D}_{0}$ at $\bar{s}$. We need to show $\left(j_{0}^{*} R^{n} f_{0 *} \overline{\mathbf{Q}}_{l}\right)_{\bar{\eta}_{s}}=H^{n}\left(\widetilde{X}_{\bar{\eta}_{s}}, \overline{\mathbf{Q}}_{l}\right)$ is an $F$-semisimple representation of $\operatorname{Gal}\left(\bar{\eta}_{s} / \eta_{s}\right)$.

Define $m$ by $n=2 m+1$ if $n$ is odd and by $n=2 m$ if $n$ is even. Let $\delta_{s} \in$ $H^{n}\left(\widetilde{X}_{\bar{\eta}_{s}}, \overline{\mathbf{Q}}_{l}(m)\right)$ be the vanishing cycle corresponding to the critical point $s$. Then $\left(\delta_{s}, \delta_{s}\right)=0$ if $n$ is odd and $\left(\delta_{s}, \delta_{s}\right)=(-1)^{m} 2$ if $n$ is even, where (, ) is the Poincaré pairing on $H^{n}\left(\widetilde{X}_{\bar{\eta}_{s}}, \overline{\mathbf{Q}}_{l}\right)$. Since $\delta_{s} \neq 0$, by [SGA7] XV 3.4, we have an exact sequence

$$
0 \rightarrow H^{n}\left(\widetilde{X}_{\bar{s}}, \overline{\mathbf{Q}}_{l}\right) \rightarrow H^{n}\left(\widetilde{X}_{\bar{\eta}_{s}}, \overline{\mathbf{Q}}_{l}\right) \stackrel{\left(, \delta_{s}\right)}{\longrightarrow} \overline{\mathbf{Q}}_{l}(m-n) \rightarrow 0 .
$$

By Lemma 2.4 below, $F_{s}$ acts semisimply on $H^{n}\left(\widetilde{X}_{\bar{s}}, \overline{\mathbf{Q}}_{l}\right)$ and with weights $\leq n$ by Deligne's theorem. On the other hand, $F_{s}$ acts semisimply on $\overline{\mathbf{Q}}_{l}(m-n)$ with weight $2(n-m)$. When $n$ is odd, we have $n<2(n-m)$. So any $F \in \operatorname{Gal}\left(\bar{\eta}_{s} / \eta_{s}\right)$ lifting $F_{s} \in \operatorname{Gal}(\bar{k}(s) / k(s))$ acts semisimply on $H^{n}\left(\widetilde{X}_{\bar{\eta}_{s}}, \overline{\mathbf{Q}}_{l}\right)$. This proves the $F$ semisimplicity in the case when $n$ is odd.

When $n$ is even, we have $\left(\delta_{s}, \delta_{s}\right)=(-1)^{m} 2$. The above exact sequence then shows that $H^{n}\left(\widetilde{X}_{\bar{\eta}_{s}}, \overline{\mathbf{Q}}_{l}(m)\right)$ is spanned by $\delta_{s}$ and $H^{n}\left(\widetilde{X}_{\bar{s}}, \overline{\mathbf{Q}}_{l}(m)\right)$. By the construction of the vanishing cycles (cf. [SGA7] XV 2.2), there exists a finite extension $K$ of $k\left(\eta_{s}\right)$ contained in $k\left(\bar{\eta}_{s}\right)$ such that $\delta_{s}$ can be defined over $K$ and hence is 
invariant under the action of $\operatorname{Gal}\left(k\left(\bar{\eta}_{s}\right) / K\right)$. Let $F$ be an element in the Weil group $W\left(k\left(\bar{\eta}_{x}\right) / K\right)$ but not in the inertia group. Then $\delta_{s}$ is invariant under the action of $F$. On the other hand, $H^{n}\left(\widetilde{X}_{\bar{s}}, \overline{\mathbf{Q}}_{l}(m)\right)$ is an invariant subspace of the action of $F$ on $H^{n}\left(\widetilde{X}_{\bar{\eta}_{s}}, \overline{\mathbf{Q}}_{l}(m)\right)$ and Lemma 2.4 shows that $F$ acts semisimply on this invariant subspace. So $F$ acts semisimply on $H^{n}\left(\widetilde{X}_{\bar{\eta}_{s}}, \overline{\mathbf{Q}}_{l}(m)\right)$. This proves the $F$-semisimplicity in the case when $n$ is even.

(ii) Note that $R^{i} f_{0 *} \overline{\mathbf{Q}}_{l}(i \neq n+1)$ are geometrically constant sheaves on $D_{0}$ by Proposition 2.1 (ii) and hence are inverse images of sheaves on $\operatorname{spec} \mathbf{F}_{q}$. Their $F$-semisimplicity follows from the induction hypothesis.

By [SGA7] XV 3.4, we have an exact sequence

$$
H^{n+1}\left(\widetilde{X}_{\bar{s}}, \overline{\mathbf{Q}}_{l}\right) \rightarrow H^{n+1}\left(\widetilde{X}_{\bar{\eta}_{s}}, \overline{\mathbf{Q}}_{l}\right) \rightarrow 0 .
$$

By the induction hypothesis and Lemma $2.4, F_{s}$ acts semisimply on $H^{n+1}\left(\widetilde{X}_{\bar{s}}, \overline{\mathbf{Q}}_{l}\right)$. So $F_{s}$ acts semisimply on $H^{n+1}\left(\widetilde{X}_{\bar{\eta}_{s}}, \overline{\mathbf{Q}}_{l}\right)$. Hence $j_{0}^{*} R^{n+1} f_{0 *} \overline{\mathbf{Q}}_{l}$ is $F$-semisimple.

Lemma 2.4. Let $Y_{0}$ be a projective scheme over $\mathbf{F}_{q}$ of dimension $n$ with only one singular point which is an ordinary double point and $\mathbf{F}_{q}$-rational. Under the induction hypothesis, $F$ acts semisimply on $H^{i}\left(Y_{0}, \overline{\mathbf{Q}}_{l}\right)$.

Proof. Let $y$ be the unique singular point of $Y_{0}$, let $\widetilde{Y}_{0}$ be the blowing-up of $Y_{0}$ at $y$ and let $p: \widetilde{Y}_{0} \rightarrow Y_{0}$ be the cannical projection. By direct computation, one can show $\widetilde{Y}_{0}$ and $p^{-1}(y)$ are smooth. By the induction hypothesis, $F$ acts semisimply on $H^{i}\left(\widetilde{Y}, \overline{\mathbf{Q}}_{l}\right)$ and on $H^{i-1}\left(p^{-1}(\bar{y}), \overline{\mathbf{Q}}_{l}\right)$, where $\bar{y}$ is the point in $Y$ above $y$. By Deligne's theorem, the weight of $F$ on $H^{i}\left(\widetilde{Y}, \overline{\mathbf{Q}}_{l}\right)$ and on $H^{i-1}\left(p^{-1}(\bar{y}), \overline{\mathbf{Q}}_{l}\right)$ are $i$ and $i-1$, respectively. Moreover, we have an exact sequence

$$
H^{i-1}\left(p^{-1}(\bar{y}), \overline{\mathbf{Q}}_{l}\right) \rightarrow H_{c}^{i}\left(\widetilde{Y}-p^{-1}(\bar{y}), \overline{\mathbf{Q}}_{l}\right) \rightarrow H^{i}\left(\tilde{Y}, \overline{\mathbf{Q}}_{l}\right) .
$$

So $F$ acts semisimply on $H_{c}^{i}\left(\widetilde{Y}-p^{-1}(\bar{y})\right)$. Since $\widetilde{Y}_{0}-p^{-1}(y) \cong Y_{0}-y, F$ acts semisimply on $H_{c}^{i}\left(Y-\bar{y}, \overline{\mathbf{Q}}_{l}\right)$. Since $H^{i}\left(\bar{y}, \overline{\mathbf{Q}}_{l}\right)=0$ for $i \geq 1$, we have an exact sequence

$$
H_{c}^{1}\left(Y-\bar{y}, \overline{\mathbf{Q}}_{l}\right) \rightarrow H^{1}\left(Y, \overline{\mathbf{Q}}_{l}\right) \rightarrow 0
$$

and $H^{i}\left(Y, \overline{\mathbf{Q}}_{l}\right)=H_{c}^{i}\left(Y-\bar{y}, \overline{\mathbf{Q}}_{l}\right)$ for $i \geq 2$. So $F$ acts semisimply on $H^{i}\left(Y, \overline{\mathbf{Q}}_{l}\right)$ for $i \geq 1$. Also $F$ acts semisimply on $H^{0}\left(Y, \overline{\mathbf{Q}}_{l}\right)=\bigoplus_{\pi_{0}(Y)} \overline{\mathbf{Q}}_{l}$.

Now let's finish the proof of Theorem 3. Since $\overline{\mathbf{Q}}_{l}[n+1]$ is a pure perverse sheaf on $\widetilde{X}_{0}$, by the Hard Lefschetz Theorem in [BBD] 5.4.10, the first Chern class $l \in H^{2}\left(\widetilde{X}_{0}, \overline{\mathbf{Q}}_{l}(1)\right)$ of the relative ample invertible sheaf for the projective morphism $f_{0}: \widetilde{X}_{0} \rightarrow D_{0}$ induces an isomorphism

$$
l^{i}:{ }^{p} R^{-i} f_{0 *}\left(\overline{\mathbf{Q}}_{l}[n+1]\right) \rightarrow{ }^{p} R^{i} f_{0 *}\left(\overline{\mathbf{Q}}_{l}[n+1]\right)(i)
$$

for all $i \geq 0$. So by [D3] 0.3, we have an isomorphism

$$
R f_{0 *}\left(\overline{\mathbf{Q}}_{l}[n+1]\right) \cong \bigoplus_{i}^{p} R^{i} f_{0 *}\left(\overline{\mathbf{Q}}_{l}[n+1]\right)[-i]
$$

and hence

$$
R f_{0 *} \overline{\mathbf{Q}}_{l} \cong \bigoplus_{i}^{p} R^{i} f_{0 *} \overline{\mathbf{Q}}_{l}[-i]
$$


So

$$
\begin{aligned}
H^{j}\left(\tilde{X}, \overline{\mathbf{Q}}_{l}\right) & \cong H^{j}\left(D, R f_{*} \overline{\mathbf{Q}}_{l}\right) \\
& \cong \bigoplus_{i} H^{j}\left(D,{ }^{p} R^{i} f_{0 *} \overline{\mathbf{Q}}_{l}[-i]\right) \\
& \cong \bigoplus_{u+v=j} H^{u}\left(D,{ }^{p} R^{v} f_{0 *} \overline{\mathbf{Q}}_{l}\right)
\end{aligned}
$$

as $\operatorname{Gal}\left(\mathbf{F} / \mathbf{F}_{\mathbf{q}}\right)$-modules. By Lemma 2.2, Lemma 2.3 and Theorem $2, F$ acts semisimply on each $H^{u}\left(D,{ }^{p} R^{v} f_{0 *} \overline{\mathbf{Q}}_{l}\right)$. So $F$ acts semisimply on $H^{j}\left(\tilde{X}, \overline{\mathbf{Q}}_{l}\right)$. This completes the proof of Theorem 3 .

\section{REFERENCES}

[BBD] A. Beilinson, J. Bernstein and P. Deligne, Faiseaux Pervers, in Analyse et Topologie sur les Espace Singuliers (I), Astérisque 100 (1982). MR 86g:32015.

[D1] P. Deligne, La Conjecture de Weil II, Publ. Math. IHES, 52 (1980), 137-252. MR 83c:14017

[D2] P. Deligne, Les Constantes des Équations Fontionnelles des Fontions L, in Modular Functions of One Variable, II, Lecture Notes in Mathematics, 349, Springer-Verlag (1973), 55-106. MR 52:8091]

[D3] P. Deligne, Décompositions dans la Catégorie Dérivée, in Motives, Proceedings of Symposia in Pure Mathematics, Vol. 55, Part 1, American Mathematical Society, Providence (1994), 115-128. MR 95h:18013

[F] L. Fu, On the Semisimplicity of Pure Sheaves, Proc. Amer. Math. Soc., 127 (1999), No. 9, 2529-2533. MR 2000a:14021

[K] N. Katz, Travaux de Laumon, Séminaire Bourbaki, 1987-1988, No. 691, Astérisque 161162 (1988), 105-132. MR 90h:14028

[L] G. Laumon, Transformation de Fourier, Constantes d'Équations Fontionnelles, et Conjecture de Weil, Publ. Math. IHES, 65 (1987), 131-210. MR 88g:14019

[ST] J.-P. Serre and J. Tate, Good Reduction of Abelian Varieties, Ann. of Math., 88 (1968), 492-517. MR 38:4488

[SGA] Séminaire de Géométrie Algébrique du Bois-Marie.

[SGA4] Théorie des Topos et Cohomologie Étale des Schémas, by M. Artin, A. Grothendieck and J.-L. Verdier, Lecture Notes in Mathematics, 269, 270, 305, Springer-Verlag, 1972, 1973. MR 50:7130 MR 50:7131, MR 50:7132

[SGA4 $\frac{1}{2}$ ] Cohomologie Étale, by P. Deligne, Lecture Notes in Mathematics, 569, Springer-Verlag, 1977. MR 57:3132

[SGA7] Groupes de Monodromie en Géométrie Algébrique, I by A. Grothendieck, II by P. Deligne and N. Katz, Lecture Notes in Mathematics, 288, 340, Springer-Verlag, 1972, 1973. MR 50:7134 MR 50:7135

Institute of Mathematics, Nankai University, Tianjin, P. R. China

E-mail address: leifu@nankai.edu.cn 